\title{
Expression of coat proteins changes during postnatal development in selected areas of the rat brain
}

\author{
Janina Borgonovo ${ }^{\mathrm{a}, 1}$, Pablo Capella ${ }^{\mathrm{a}, 1}$, Alicia Seltzer ${ }^{\mathrm{c}, 1}$, Miguel A. Sosa ${ }^{\mathrm{a}, \mathrm{b}, *, 1}$ \\ a Laboratorio de Biología y Fisiología Celular “Dr. Francisco Bertini”, Instituto de Histología y Embriología, FCM, Universidad Nacional de Cuyo, Mendoza, Argentina \\ b Instituto de Ciencias Básicas-Universidad Nacional de Cuyo, Mendoza, Argentina

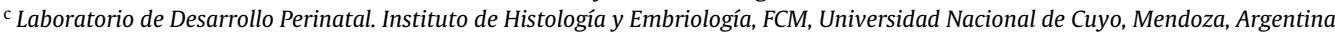

\section{A R T I C L E I N F O}

\section{Article history:}

Received 7 September 2011

Received in revised form 3 January 2012

Accepted 17 January 2012

\section{Keywords:}

Clathrin coated vesicles

Adaptins

Endocytosis

Intracellular transport

Brain development

\begin{abstract}
A B S T R A C T
It is well known that clathrin-mediated endocytosis is crucial for the normal functioning and integrity of neurons in the central nervous system. In this study we attempted to correlate the expression of coat proteins with development in different areas of rat brain. By Western blot, we studied the expression of AP-2, GGA1 and GGA2 in striatum, cerebellum, brain stem, cerebral cortex and hippocampus of newborn rats and during post-natal development; $5,15,30,60,90$ or 150 days after birth. We observed that the expression of the $\alpha 2$ subunit of AP-2 increased substantially between the 15th and 30th day after birth in all areas studied, excepting the cerebellum and cortex. On the other hand, the expression of the $\alpha 1$ subunit does not change significantly during the development in any of the areas under study. We also noted that the expression of the $\mu 2$ subunit did not follow the pattern of $\alpha 2$ during development. In general terms, the expression of GGA1 and GGA2 followed a similar pattern to that of AP-2, although these proteins increased significantly in the cerebral cortex from the 15 th day after birth. Moreover, presenilin1 , a protein associated with aging and neurodegeneration, shows an expression pattern similar to coat proteins in the striatum and cortex. These results suggest that proteins that conform the intracellular transport machinery in the brain cells seems to accompany development, according to the maturation of the different brain areas.
\end{abstract}

(C) 2012 ISDN. Published by Elsevier Ltd. All rights reserved.

\section{Introduction}

Vesicle-mediated transport is one of the main ways to carry material from one area to another within the cell, or in the exchange with the extracellular environment or with other cells (Conner and Schmidt, 2003). In the central nervous system, the clathrinmediated transport appears crucial for the proper functioning and maintenance of the integrity of neurons (Hirst and Robinson, 1998). Thus, the study of proteins that form the coat of the vesicles could give us some clues about the conditions of the cells on the different areas of the brain. These clathrin-coated vesicles (CCVs) are involved in endocytosis and transport from the trans-Golgi network (TGN) to endosomes (reviewed by Kirchhausen, 1999). Although clathrin is a major component of the coat of the vesicles, from the functional standpoint adaptins (APs) play a key role in the formation of the coat, as they confer selectivity. Four families of APs (AP

\footnotetext{
* Corresponding author at: Instituto de Histología y Embriología, FCM, Universidad Nacional de Cuyo, Mendoza-Argentina. Tel.: +54 261 413500x2677; fax: +54 2614494117

E-mail address: msosa@fcm.uncu.edu.ar (M.A. Sosa).

1 Equal contribution.
}

1-4) have been described so far, of which AP-2 is specific to the CCVmediated endocytosis and plays an important role in membrane homeostasis of nerve terminals (Granseth et al., 2007; Granseth and Lagnado, 2008). AP-2 is a heterotetramer composed of two major subunits $(\alpha, \beta 2)$, a medium subunit ( $\mu 2)$ and a small subunit $(\delta 2)$. Interestingly, in nervous tissue two isotypes of $\alpha$ subunit have been described, $\alpha 1$ and $\alpha 2$, but the co-existence of these isotypes is not yet well understood. Other proteins functionally related to CCVs have been identified, such as the family of the Golgi-localised, gamma-ear containing, ARF-binding proteins (GGA1-3) (Robinson and Bonifacino, 2001; Hirst et al., 2007; Zhang et al., 2007). These proteins, along with a myriad of accessory proteins (Owen et al., 1999; Collins et al., 2002) form a complex machinery, leading to the formation of the CCVs. In addition, some proteins and lipids in membranes are the partners for the recruitment of coat proteins.

It has been shown that the dynamics of formation of CCVs changes during the growth of neurons in primary cultures, to the point that the cycle of assembly-disassembly of the coat is faster in young neurons (Blanpied et al., 2002). Furthermore, it seems that changes in the dynamics of uptake of molecules by endocytosis plays a considerable role in neuronal aging (Benzi and Moretti, 1995; Samson and Nelson, 2000), as well as in age-related diseases such as Parkinson's and Alzheimer's disease (Herbert et al., 1994; 
A.

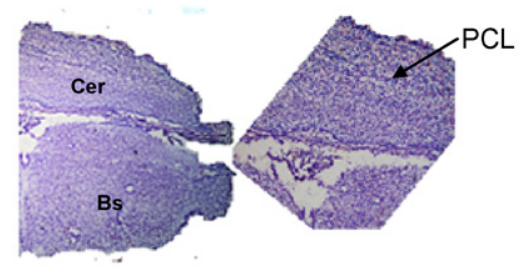

$4 \mathrm{x}$

C.

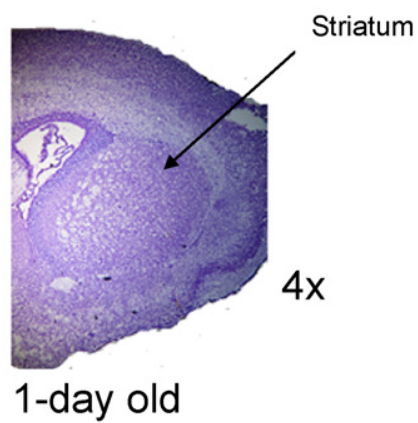

B.

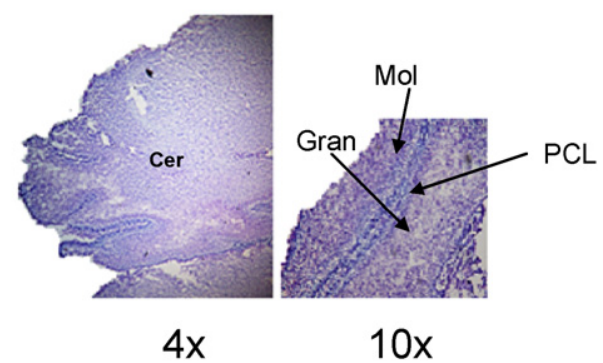

D.

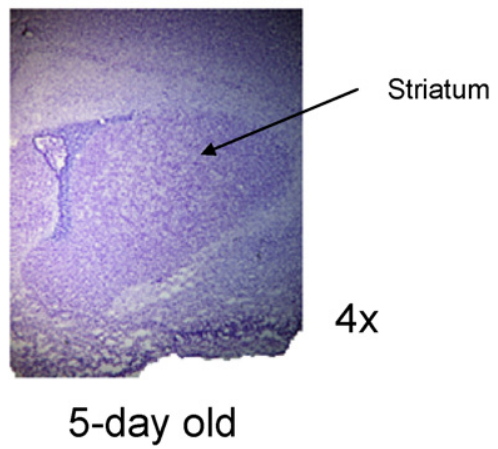

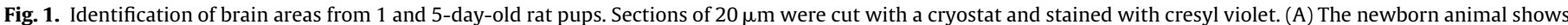

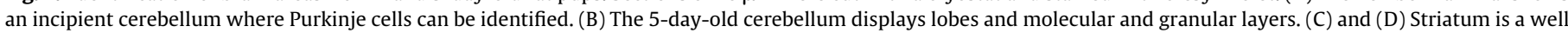

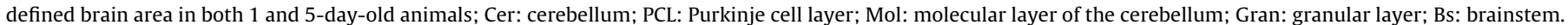

Jellinger, 1999; Gaidarov and Keen, 1999; Foley and Riederer, 2000; Double et al., 2000; Halliwell, 2001).

In recent years, studies on endocytosis in nerve terminals became a priority, as it could have implications for neuromodulation and neuronal growth (Nagappan and Lu, 2005; Yamashita et al., 2010; Hines et al., 2010; Assaife-Lopes et al., 2010). The importance of the endocytic machinery in the maintenance of neuronal integrity is also supported by evidence showing changes in the expression of AP-2 and loss of its affinity for membranes in certain brain areas when subjected to the effect of excitotoxic drugs (Borgonovo et al., 2009).

This study attempted to determine whether the intracellular transport machinery accompanies neuronal development in different areas during postnatal development. To address this, by applying the immunoblot and immunohistochemistry methodologies, we studied the expression of proteins involved in the intracellular transport, in the cerebral cortex, Corpus striatum, hippocampus, cerebellum and brain stem from newborn rats and at different ages after birth.

\section{Materials and methods}

\subsection{Subjects}

Wistar rats used in this study were newborn (less than 1 day), 5, 15, 30, 60, 90 and 150 days after birth. The different brain areas from newborn (24 pups) and 5-15-day-old animals were pooled into three independent groups, $(n=3)$. Older animals (age 30-150 days) were processed individually (three animals for each age). The newborn were sacrificed $3 \mathrm{~h}$ after birth, and older pups were kept with their mothers and siblings until sacrifice. The rats aged 15-150 days were kept under controlled temperature, humidity and light-dark cycles with food and water available ad libitum. All experiments were carried out in accordance with the rules set in the National Institute of Health guide for the care and use of laboratory animals and the local animal-ethic instructions set by the CICUAL (Comité Institucional para el cuidado y uso de Animales de Laboratorio) de Argentina.

\subsection{Materials and reagents}

The monoclonal IgG against $\alpha$-adaptin (AP-2) (Sigma Chemicals, St. Louis, MO, USA, A 4325) was prepared in mouse against AP-2 adaptor polypeptides from bovine brain, and it reacts with the 105 and $110 \mathrm{kDa} \alpha$-subunits of adaptor complex AP2 in bovine and rats. The mouse monoclonal antibody against the $\mathrm{N}$-terminus (R11-29; amino acids 11-29) of $\mu 2$ subunit was kindly provided by Dr. Bonifacino (Bethesda, USA). The goat polyclonal IgG against GGA-1 was purchased from Santa Cruz (sc-23261) raised against an epitope mapping at the N-terminus of human GGA1, and it recognizes a unique band arround $67 \mathrm{kDa}$. The goat polyclonal IgG against GGA2 (Santa Cruz, sc-19326) was raised against an epitope

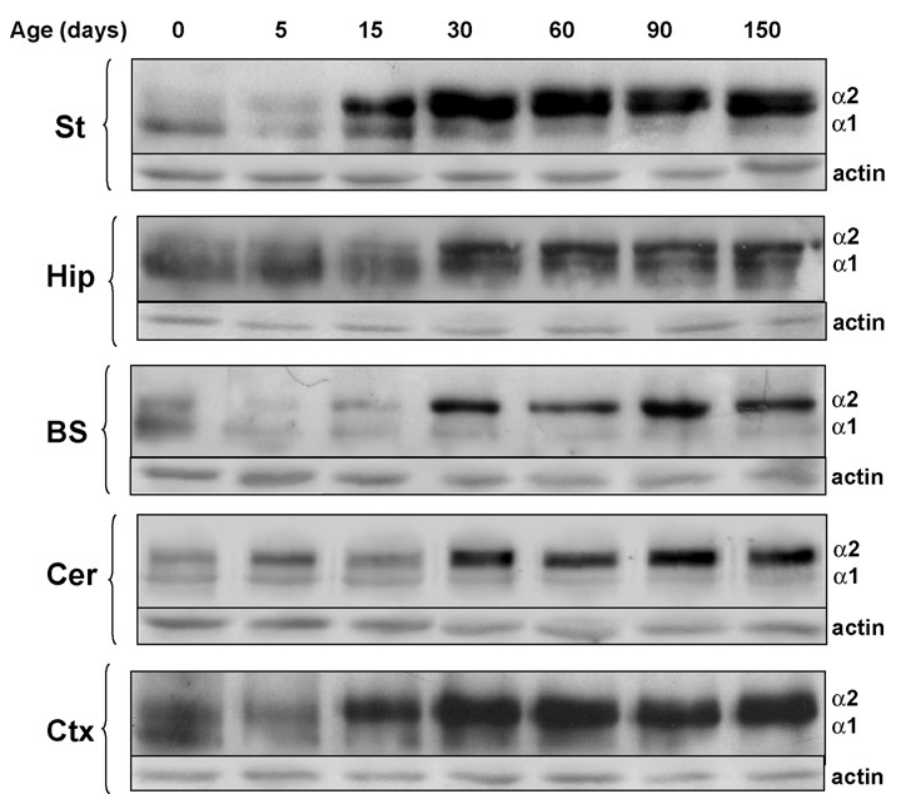

Fig. 2. Immunoblot analysis of AP-2 ( $\alpha$ subunits) in different areas of rat brain. Proteins $(45 \mu \mathrm{g})$ from homogenates of each brain area, at the indicated ages, were subjected to electrophoresis, transferred to nitrocellulose membranes and tested for $\alpha 1$ and $\alpha 2$ subunits of AP-2 with the specific monoclonal antibody that recognize both isoforms. The image shows a representative immunoblot from three independent experiments at each age, as detailed in Section 2. The $\alpha 1$ and $\alpha 2$ isoforms of AP-2 are indicated in the figure. St: striatum; Hip: hippocampus; BS: brainstem; Cer: cerebellum; Ctx: cerebral cortex. 

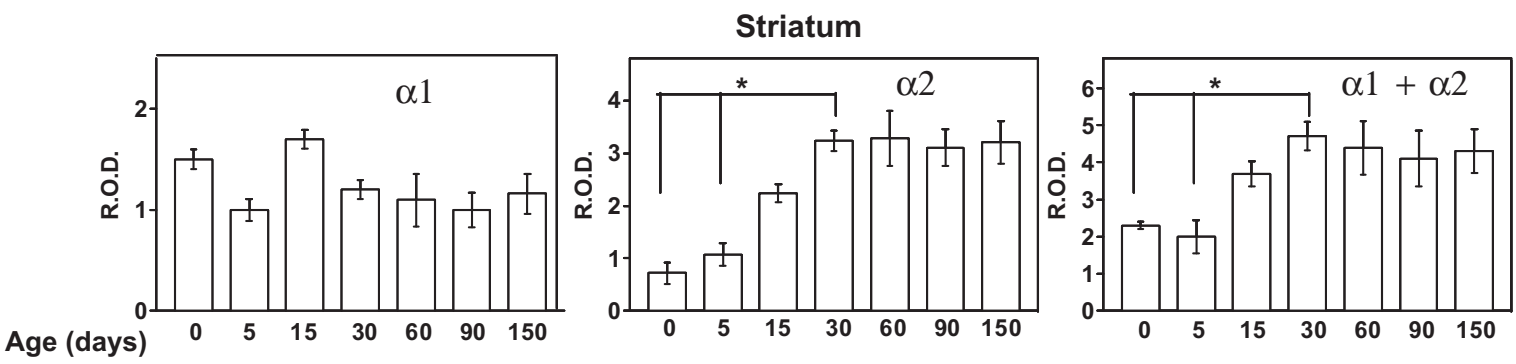

Hippocampus
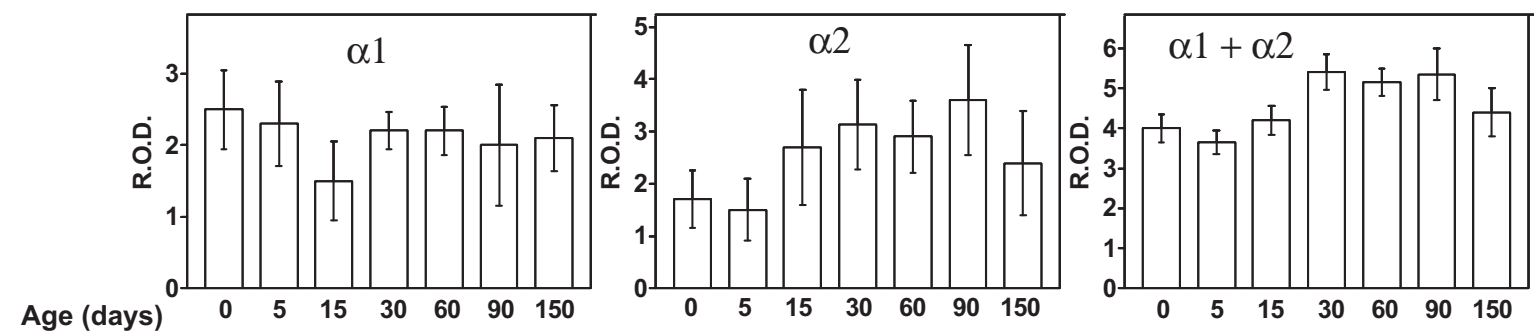

Brainstem
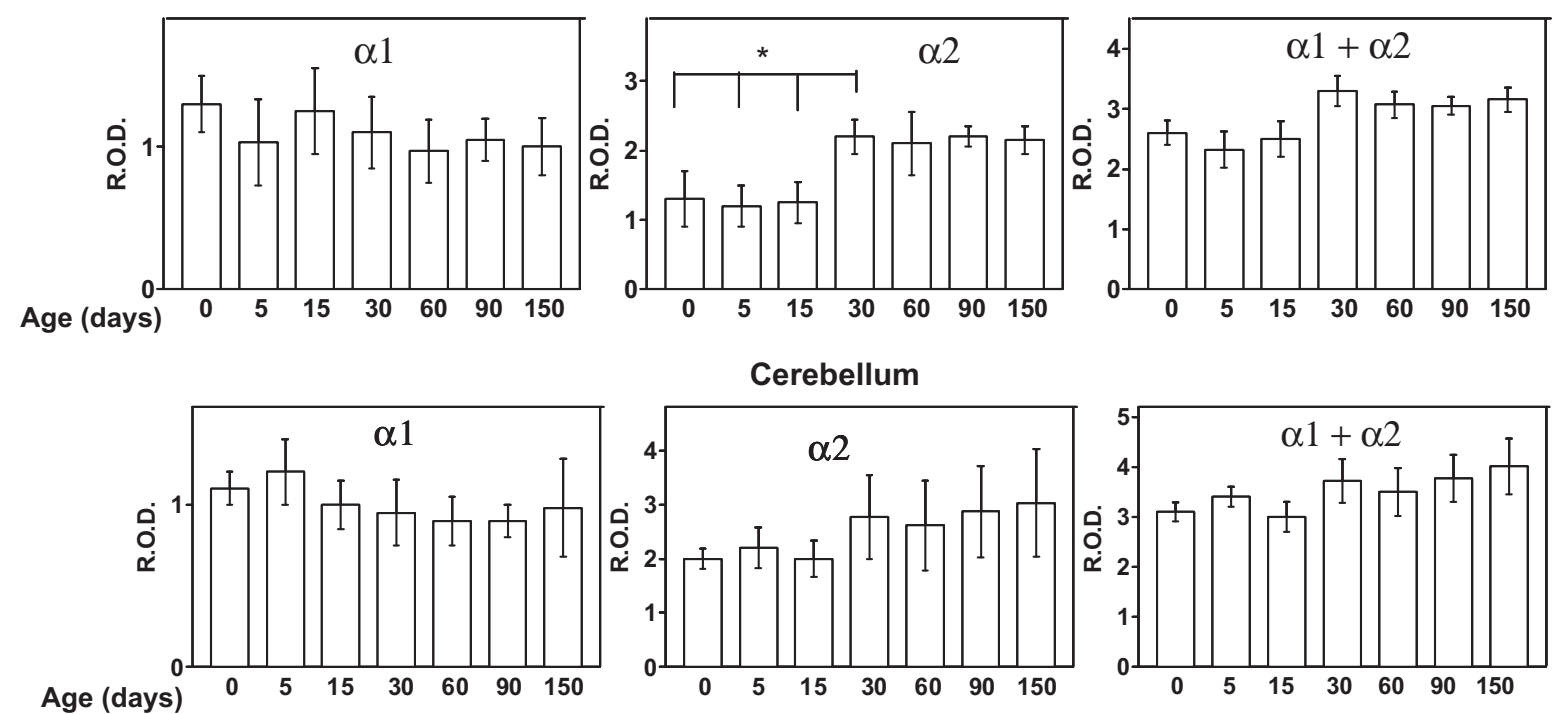

Cerebellum
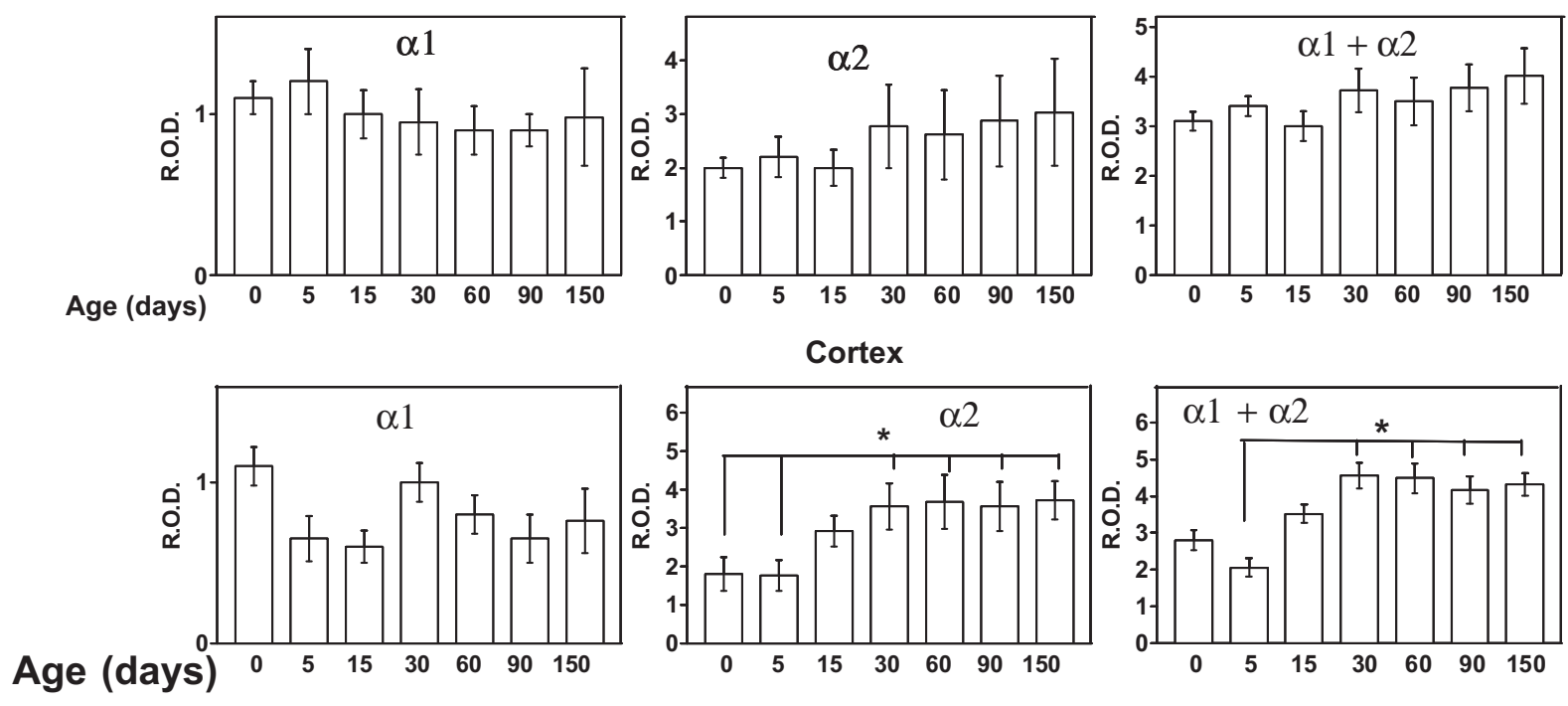

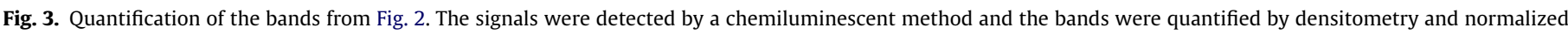

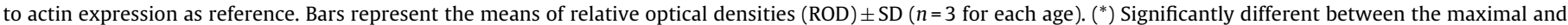
minimal values $(P<0.05)$.

mapped within an internal region of the human GGA2 that reacts with a unique band of $85 \mathrm{kDa}$ in rats. The antibody against presenilin-1 (PS-1) (Sigma Chemicals, St. Louis, MO, USA, P7854), was produced in rabbit (IgG fraction), raised against a synthetic peptide (17 aminoacids) corresponding to the C-terminal of human PS- 1 and reacts against a band of $48 \mathrm{kDa}$ from rat brain (full length t protein). The anti-mouse IgG (Fc specific) biotin conjugate (B9904), anti-rabbit IgG (whole molecule) biotin conjugate (B8895), anti-goat IgG (whole molecule) biotin conjugate (A9294), and avidin-peroxidase conjugate (A7419) were all purchased from Sigma Chemicals. Chemiluminescent reagents were from Pearce (Rockford, IL, USA).

\subsection{Sample processing}

Rats were sacrificed by decapitation and the following brain areas were identified and dissected: cerebral cortex, striatum (bilateral), hippocampus (bilateral), brainstem and cerebellum. Tissues were weighed and homogenized with a glass homogenizer and Teflon pestle, in $20 \mathrm{mM}$ HEPES buffer $\mathrm{pH} 7.4(1: 3 \mathrm{w} / \mathrm{v})$, containing $0.25 \mathrm{M}$ sucrosa, 2 mM EDTA and a mixture of protease inhibitors ( $1 \mathrm{mM}$ PMSF, $10 \mathrm{mM}$ $\mathrm{NaF}, 1 \mu \mathrm{M}$ pepstatin, $100 \mu \mathrm{M}$ leupeptin, $0.1 \mathrm{TIU} / \mathrm{ml}$ aprotinin) purchased from Sigma Chemicals. The homogenates were then centrifuged at $800 \times g$ for $10 \mathrm{~min}$, and the post-nuclear supernatants were recovered and stored at $-20^{\circ} \mathrm{C}$ up to use. 
A.
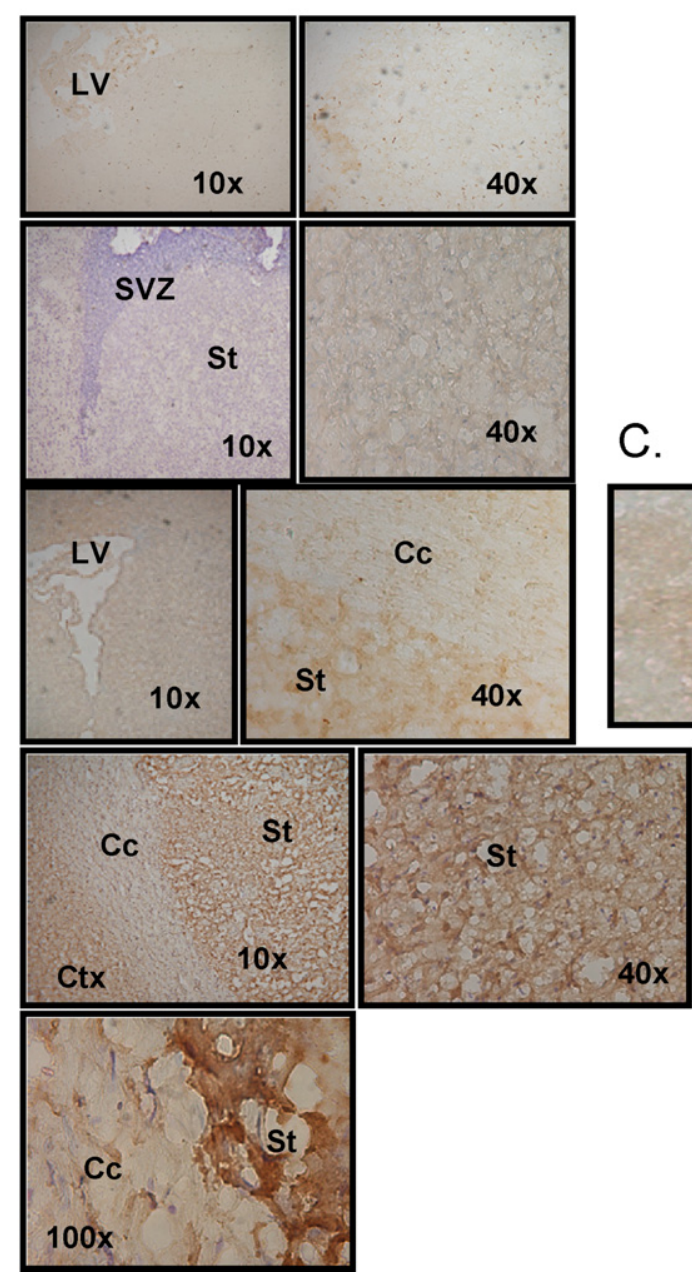

B.

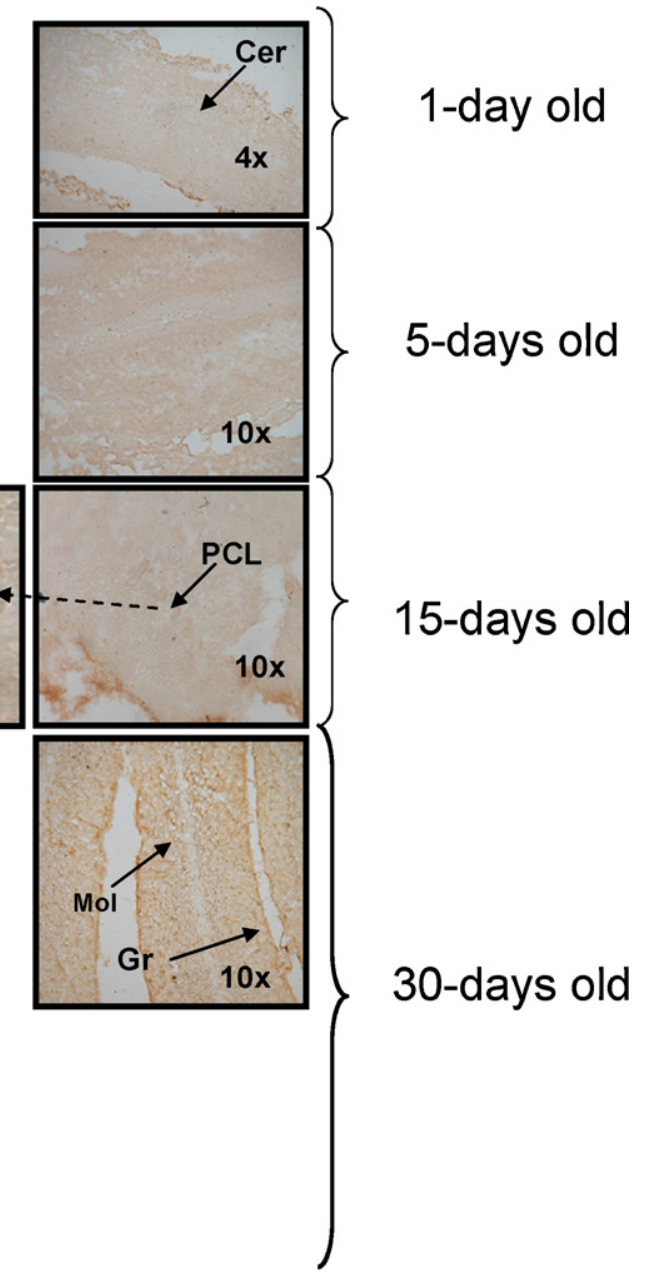

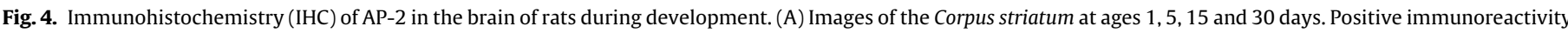

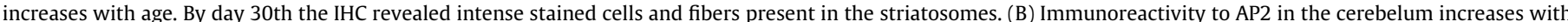

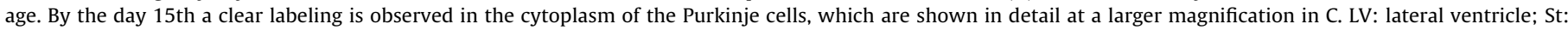

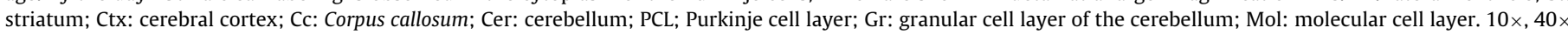
and $100 \times$ : magnification of the microscope objective.

\subsection{Electrophoresis and immunoblotting}

All procedures were carried out according to Alberdi et al. (2005). Briefly, $45 \mu \mathrm{g}$ of protein from each sample were analyzed on $8 \%$ acrylamide gels (SDS-PAGE), and then electrotransfered onto nitrocellulose membranes and processed for detection of the proteins under study: $\alpha 1-\alpha 2(1: 5000)$ and $\mu 2$ (1:1000) subunits of AP-2, GGA1 (1:1000), GGA2 (1:1000), and PS-1 (1:500), with the corresponding monoclonal antibodies. After incubating with biotin-conjugated antibody and subsequently with avidin-peroxidase, the specific bands were detected by a chemiluminescence method, and the bands were quantified from the films (Kodak X-Omat) by densitometric scanning and analyzed with the Scion Image program. The values of density were normalized for equal loadings in the gels by comparing with actin expression as follows: protein O.D. (AP-2, GGAs or PS-1)/actin O.D. $\times 1.2$ (Correction factor to approximate values to 1$)$.

\subsection{Immunohistochemistry}

Animals were deeply anesthetized with chloral hydrate, perfused intracardially with a washing solution $(0.8 \% \mathrm{NaCl}, 0.8 \%$ sucrose, $0.4 \%$ glucose $)$ and then with $4 \%$ paraformaldehyde in $0.01 \mathrm{M}$ phosphate buffer $\mathrm{pH} 7.3$ and $0.35 \mathrm{M} \mathrm{Na}_{2} \mathrm{SO}_{3}$. The brains were cryoprotected and stored at $-70^{\circ} \mathrm{C}$. Slices $(20 \mu \mathrm{m})$ were mounted on gelatin- alum-chrome coated slides and treated for antigen retrieval by placing the sections on $10 \mathrm{mM}$ sodium citrate buffer, $\mathrm{pH} 6.0$ at $95^{\circ} \mathrm{C}$, incubated in blocking solution ( $10 \mathrm{mM}$ phosphate buffer, $0.9 \% \mathrm{NaCl}, \mathrm{pH} 7.4$, with $0.1 \%$ Triton $\mathrm{X}-100$, $1 \%$ goat serum and $1 \%$ bovine serum albumin). The primary antibody was a rabbit monoclonal anti $\alpha$-adaptin (Sc-10761, from Santa Cruz Biotechnology, Inc.) 1:100, diluted in $10 \mathrm{mM}$ phosphate buffer, $0.9 \% \mathrm{NaCl}$, Triton $0.1 \%$. The slides were then incubated with biotin-conjugated anti-rabbit IgG (Sigma, B8895, 1:200) for $2 \mathrm{~h}$ at room temperature. Subsequent incubation was carried out with HRP-conjugated ExtrAvidin $^{\circledR}$ (Sigma, Mouse ExtrAvidin ${ }^{\circledR}$ Peroxidase Staining Kit, 1:100) and diaminobenzidine (DAB) $/ \mathrm{H}_{2} \mathrm{O}_{2}$ for color development. As a negative control, the primary antibodies were omitted and no specific labeling was identified in these sections. Slices were counterstained with cresyl violet, mounted, dehydrated and coverslipped with Bálsamo de Canadá (Biopack). Sections were photographed with a light microscope (NikonAlphaphot-2 and the Software ACT2U).

\subsection{Other procedures}

Protein concentration was measured according to Lowry et al. (1951).

\subsection{Statistics}

The data obtained were subjected to Tukey's Multiple Comparisons Test and the level of significance was set at $P \leq 0.05$.

\section{Results}

\subsection{Identification of brain areas in newborn and 5-day-old rat pups}

As it is known, rat offspring are born before total completion of several nervous system organs, but around birth most of them are delineated. In order to corroborate the presence of brain area boundaries in very young animals we obtained $20 \mu \mathrm{m}$ sections of 


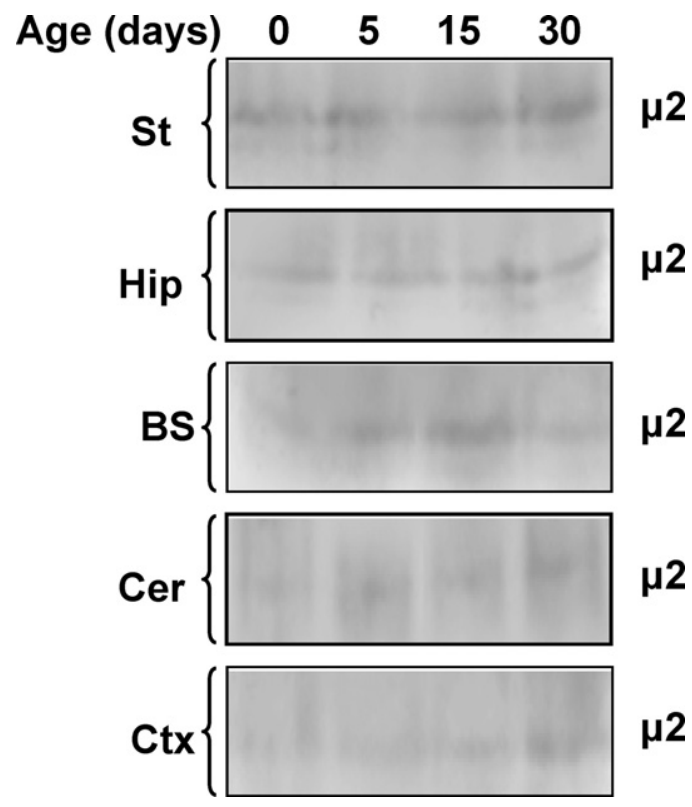

Fig. 5. Immunoblot analysis of $\mu 2$ subunit in different areas of rat brain. Proteins (45 $\mu \mathrm{g}$ ) from homogenates of each brain area, at the indicated ages, were subjected to electrophoresis, transferred to nitrocellulose membranes and tested for $\mu 2$ subunit of AP-2 with the specific monoclonal antibody. St: striatum; Hip: hippocampus; BS: brainstem; Cer: cerebellum; Ctx: prefrontal cortex.

the brains containing the areas of interest, stained them with cresyl violet and identified their boundaries with the help of a low magnification $(4 \times)$ microscopic objective (Fig. 1).

\subsection{Expression of AP-2 during postnatal development}

It was observed that expression of AP-2 changes specifically during perinatal development according to the brain area (Fig. 2). Interestingly, the expression of the $\alpha 2$ subunit is almost undetectable in the striatum of newborn and up to 5-day-old rats. However, on the 15th day, the expression of this protein increases substantially, reaching a maximum around the 30th day. This level is maintained during 150 days after birth. In contrast, $\alpha 1$ isoform does not change significantly throughout perinatal development. A similar trend was observed in hippocampus, prefrontal cortex and brainstem (Fig. 3), meanwhile, in the cerebellum, both $\alpha$ isoforms of AP-2 show little change during postnatal development. However, when both $\alpha$ isoforms were quantified together as representative of AP-2 the differences between early and late ages were significant only in the striatum and cortex (Fig. 3). The expression of actin was used as load control (Fig. 2). Immunohistochemistry of $\alpha$ subunits of AP-2 confirmed the results found by immunoblot (Fig. 4). On the other hand, the expression of the $\mu 2$ subunit did not vary between the newborn and the other postnatal ages, in any of the studied areas (Fig. 5). This was somehow expected, as this subunit interacts indistinctly with both, $\alpha 1$ and $\alpha 2$ isoforms of AP-2.

\subsection{Expression of GGA1 and GGA2 during rat postnatal development}

In general, it was noted that GGA1 expression is very low at the early stages of development, and tends to increase around the 30th day after birth (Fig. 6). However, only in the hippocampus and prefrontal cortex the differences between younger and older ages were significant (Fig. 6B). As with GGA1 and AP-2, the expression of GGA2 is low in newborn and 5-day-old pups, and tends to increase around the day 15 th in the striatum and cortex, and by the day 30th in the hippocampus (Fig. 7). Meanwhile, in brainstem and cerebellum the expression of this protein does not change significantly during development (Fig. 7).

\subsection{Expression of presenilin during rat postnatal development}

We set as objective to explore whether presenilin (PS-1), a protein related to aging and neurodegeneration, evolves similarly to the coat proteins. By studying the distribution of PS- 1 in brain tissue during development in comparison with the evolution of coat proteins, we attempted to determine whether the endocytic processes and the vesicular transport are developmentally associated. PS-1 is not similarly distributed among the areas studied, since the expression of this protein is very high in the striatum compared to other areas, even from the 15th day of age, whereas in the cortex reaches the maximum around the 30th day after birth (Fig. 8A). In turn, the expression of PS-1 (full lenght $\sim 48 \mathrm{kDa}$ ) varies differently in each brain area during development; in the striatum followed a similar trend to that observed for coat proteins, whereas in the hippocampus, cerebellum and cortex, the expression of this protein remains unchanged during postnatal development. A striking case is the brainstem, where PS1 reaches a maximum on the 30th day after birth, and then it tends to decline (Fig. 8B).

\section{Discussion}

Coated vesicles are the main vehicles to transport macromolecules between cellular compartments. In the central nervous system (CNS), it is thought that clathrin-coated vesicles (CCVs) are actively involved in maintaining the integrity and neuronal plasticity. Among the large number of coat proteins, the adaptor complexes (APs) confer specificity to the CCVs, so that AP-2 is unique to the CCVs in the receptor-mediated endocytosis in most cell types. Recent findings have suggested that clathrin-mediated endocytosis might be altered in neurodegenerative processes, as well as in the mechanisms of excitotoxicity caused by drugs ( $\mathrm{Wu}$ and Yao, 2009; Borgonovo et al., 2009). However, it is not known whether these alterations in the endocytic machinery are cause or consequence, or both, of neural disorders. AP-2 complexes consist of two major subunits, $\alpha$ and $\beta 2$, a midsized subunit $\mu 2$, and a small subunit $\delta 2$. In the nervous system, two isoforms of $\alpha$ subunits have been described, $\alpha 1$ and $\alpha 2$ (or $\alpha \mathrm{A}$ and $\alpha \mathrm{C}$ adaptin respectively) (Ball et al., 1995; Hirst and Robinson, 1998), but co-existence of both forms have not yet been explained. We believe that the $\alpha 1$ subunit would be involved in clathrin-mediated endocytosis in the cell body, whereas $\alpha 2$ might be specific for vesicles of nerve terminals. This suggestion is based on the fact that $\alpha 1$ is common to all tissues, while $\alpha 2$ is mostly found in the CNS neurons (Ball et al., 1995).

In this study, we found that the expression of both subunits varies differently during postnatal development in some areas of rat brain. In striatum, $\alpha 1$ can be detected even at early ages, whereas $\alpha 2$ is highly expressed on the 15 th day after birth. A similar trend was observed in other areas of the brain, such as hippocampus, brainstem and cortex. However, in the hippocampus and cortex, the appearance of $\alpha 2$ was observed from the day 30 after birth onwards. From these observations, we might assume that the period between the 15th and 30th day after birth (depending on the area) could be crucial for the establishment and/or expansion of neural networks, and for new synapses during development of nervous tissue. Although the subunit $\mu 2$ did not follow the pattern of $\alpha 2$, this apparent discrepancy could be explained by the ability of $\mu 2$ to interact indistinctly with both isoforms of $\alpha$ subunit. Other proteins involved in intracellular transport mediated by vesicles, such 
A Age (days)

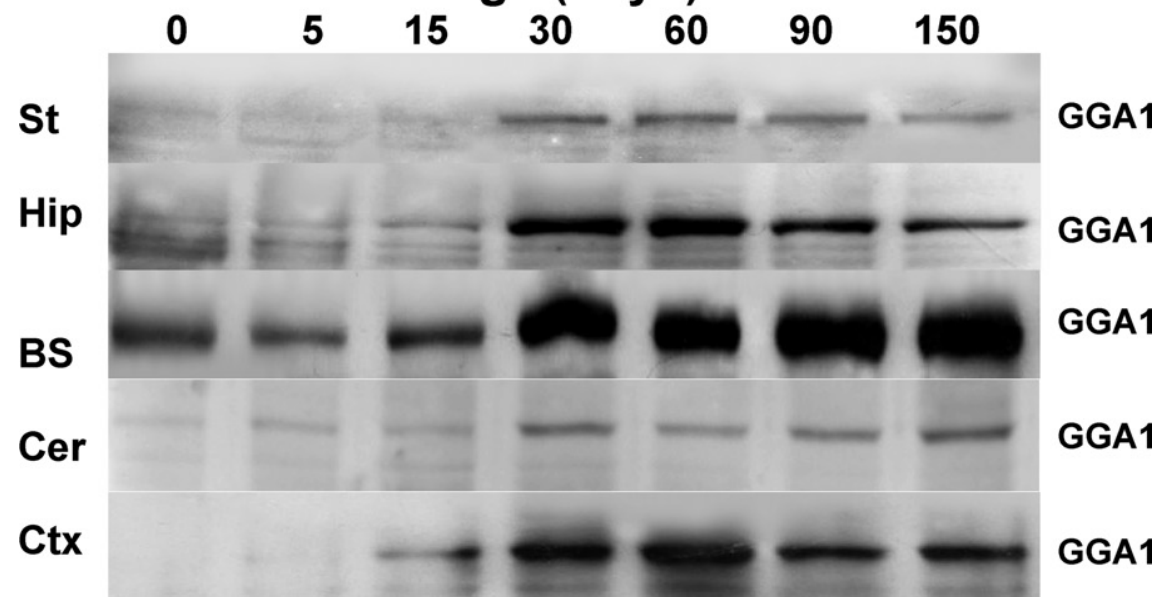

B
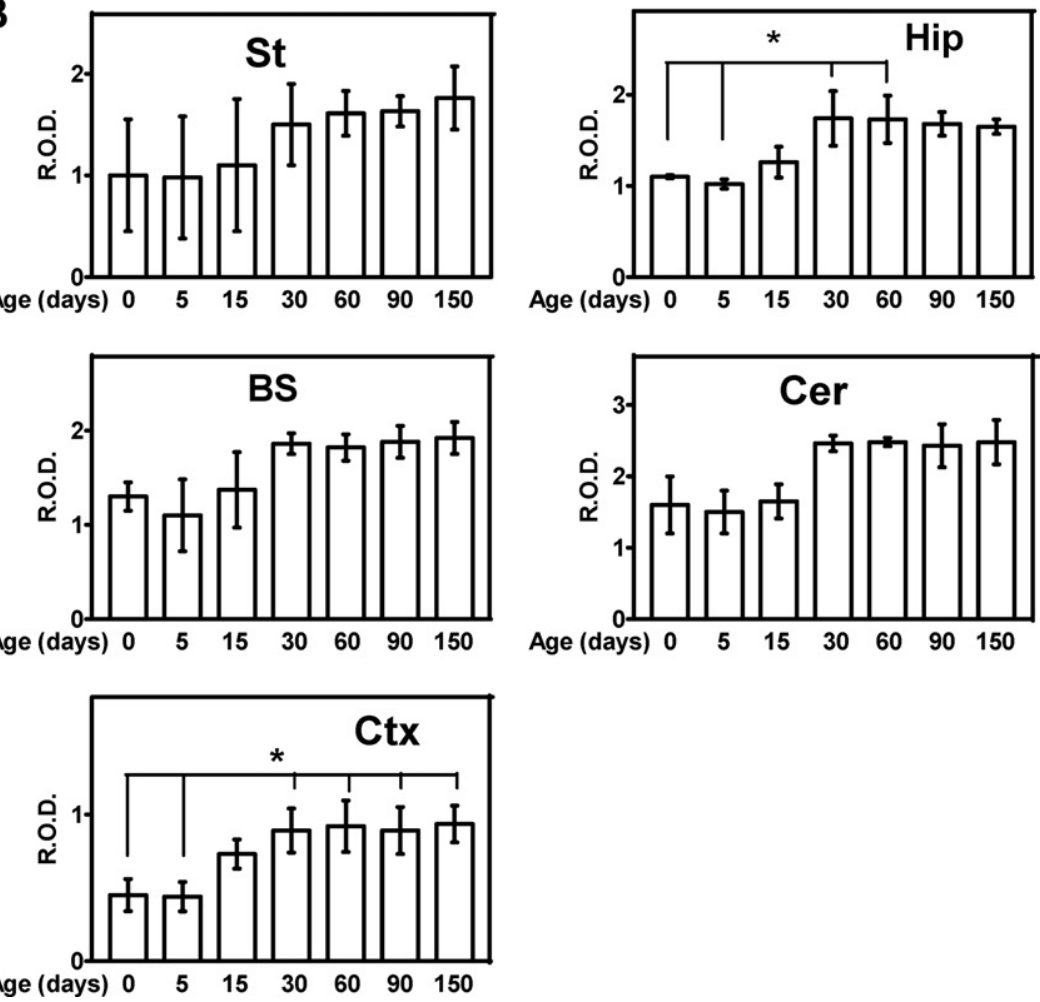

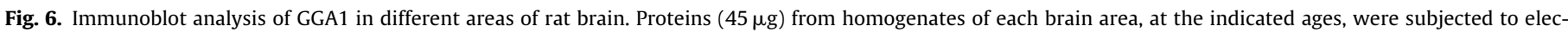

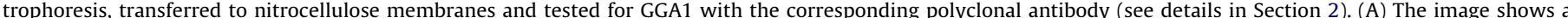

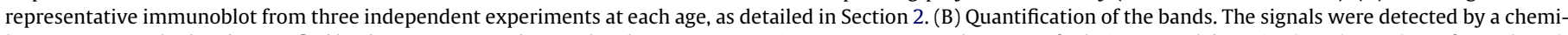

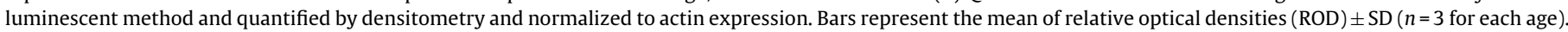
$\left({ }^{*}\right)$ Significantly different between the maximal and minimal values $(P<0.05)$. St: striatum; Hip: hippocampus; BS: brainstem; Cer: cerebellum; Ctx: cerebral cortex.

as GGA1 and GGA2 also vary in different brain areas during postnatal development, accompanying somehow the evolution of the $\alpha 2$ subunit.

It is known that the striatum consists mainly of medium spiny neurons ( $95 \%$ ) and only $10 \%$ of interneurons (reviewed by Jain et al., 2001). The GABAergic interneurons modulate the cortical input, and mediate inhibition of striatal medium-sized spiny projection neurons. Recently, it has been shown that interneurons are under the cortical control during the first days of life, and they mature considerably in the striatum of rats between $12-19$ postnatal days (Chesselet et al., 2007). It is also likely that the development and maturation of the medium spiny neurons may occur in these critical days of development, in parallel with the interneurons and expansions of neurons in the cortex.

A similar tendency was observed in the hippocampus, indicating that the endocytic machinery could reach the maximum development around 30 days after birth. As a precedent, both AP-180 and CALM, other proteins in CCVs that play a critical role in establishing neuronal polarity, are essential for the growth of dendrites and axons in hippocampal neurons (Bushlin et al., 2008). It has been shown that by the end of embryonic development and continuing into the postnatal period in the murine, there is a secondary 

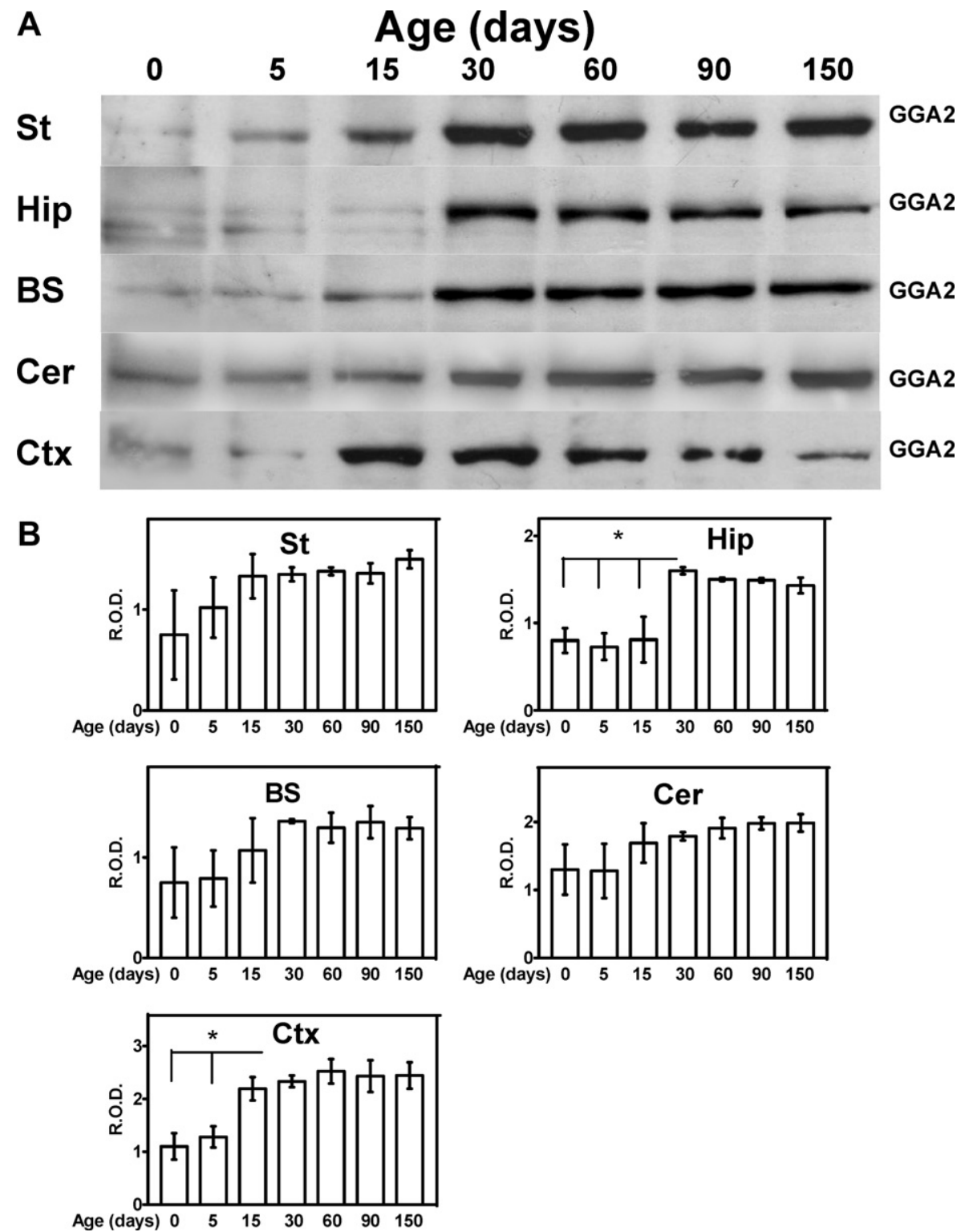

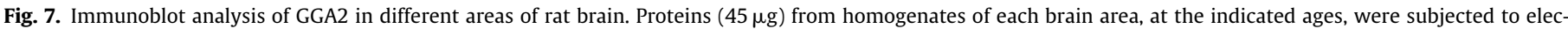

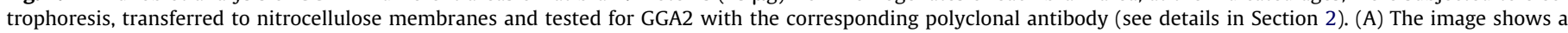

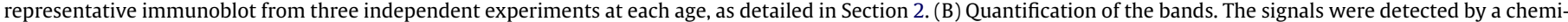

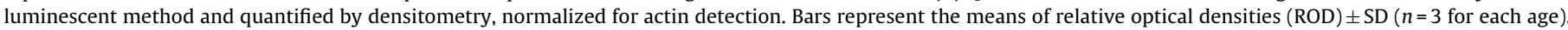
$\left({ }^{*}\right)$ Significantly different between the maximal and minimal values $(P<0.05)$. St: striatum; Hip: hippocampus; BS: brainstem; Cer: cerebellum; Ctx: prefrontal cortex.

neurogenesis in the subventricular area, in the dentate gyrus and in the cerebellar external lamina, being mainly restricted to the olfactory bulb, hippocampus and cerebellar cortex (Abrous et al., 2005).

The fact that most granule cells are generated during the first week after birth and that a massive migration of neuronal precursors towards the olfactory bulb and cortical and subcortical areas occurs around the third week after birth could be consistent with the evolution of the endocytic machinery observed in this study. In cerebellum, the expression of the $\alpha 2$ subunit was different from other areas, as this protein is expressed already at birth and remains almost unchanged throughout life. Increases in the expression of the $\alpha 2$ subunit during postnatal development were corroborated by immunohistochemistry, noting a more intense staining at later ages (30th day). Not only the endocytic machinery evolves during development in some brain areas, but also the intracellular transport mediated by vesicles could increase around 15-30 days of age.
This assumption arises from the fact that GGAs expression (GGA1 and GGA2) tended to increase between 15 and 30 days after birth in the areas studied. The increase of the coat protein expression in the cerebellum seems to initiate as early as postnatal day 17 , when granule cell proliferation and migration decline in this area and from postnatal day 30 onward, after the end of the histogenetic processes (Altman, 1982) in the rat.

Presenilin 1 (PS-1) is an integral component of the complex of high molecular weight gamma-secretase, which participates in the cleavage of type I membrane proteins such as amyloid precursor protein. Recent studies have shown that proteolytic fragments of PS-1 are enriched in clathrin-coated vesicles from somatodendritic membranes (Efthimiopoulos et al., 1998), suggesting a regulatory role of presenilin in the constitutive secretion and endocytosis. We confirmed that the expression of PS- 1 changes during postnatal development in different areas of the brain, although these changes were dissimilar from one area to another. Even though the 
A Age (days)

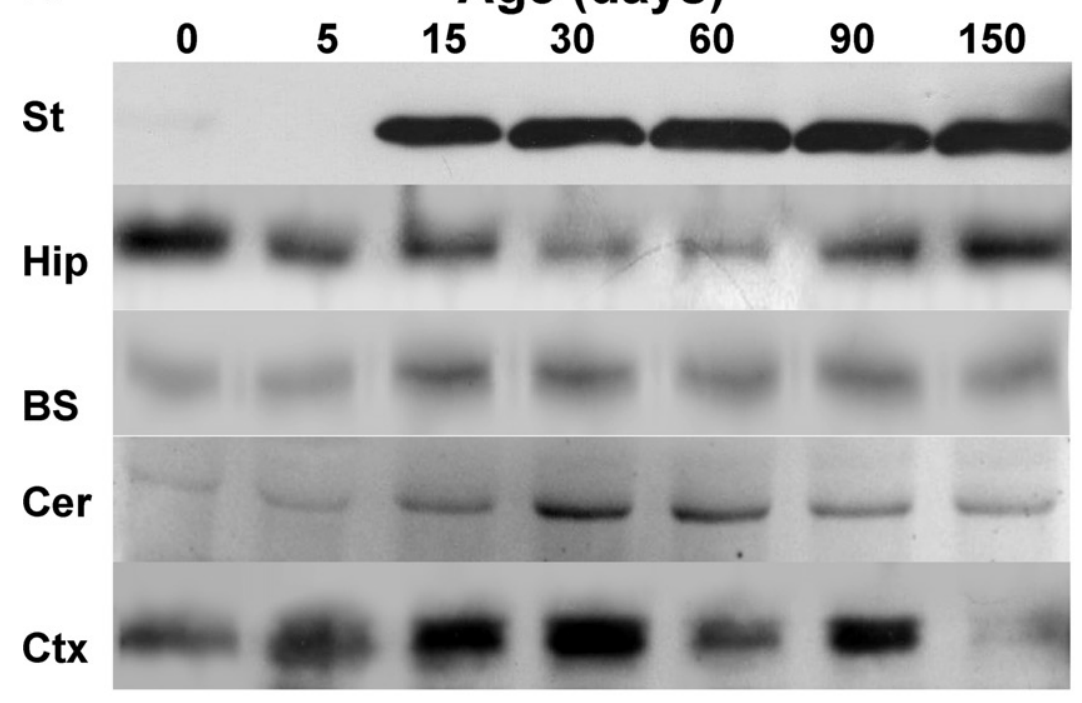

$48 \mathrm{kDa}$ $48 \mathrm{kDa}$

\section{$48 \mathrm{kDa}$}

$48 \mathrm{kDa}$

B
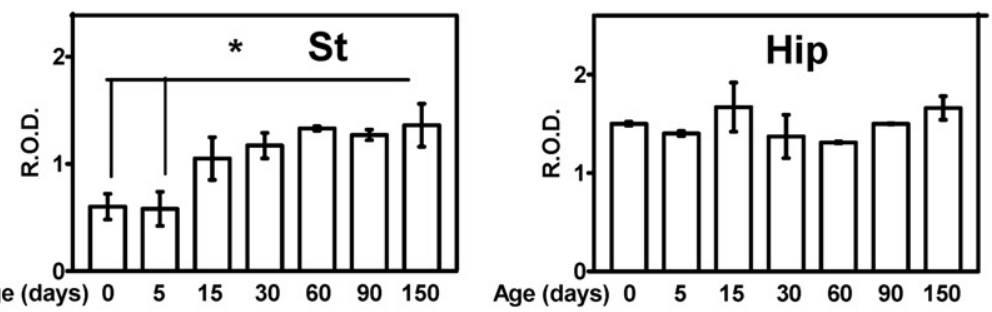

$48 \mathrm{kDa}$
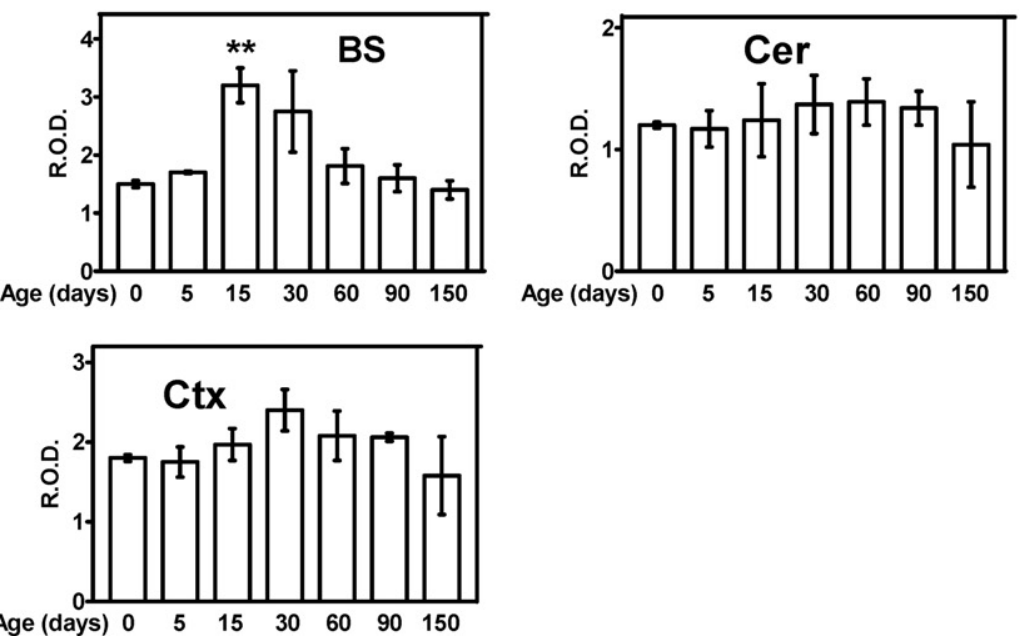

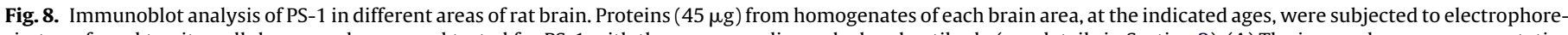

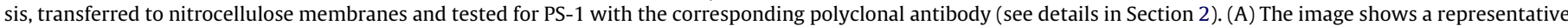

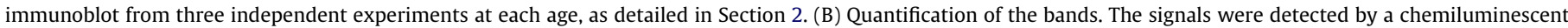

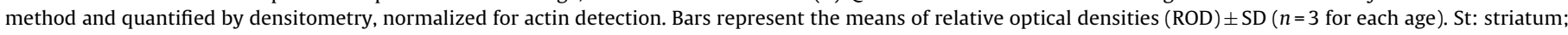

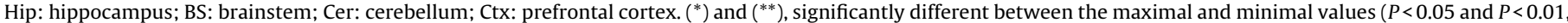
respectively).

expression of PS-1 in the cerebellum and hippocampus during postnatal development was similar to that observed by other authors (Ribaut-Barassin et al., 2003), we found no correlation with the evolution of AP-2 nor GGAs at all ages studied However, in the striatum the expression of PS-1 coincided with the appearance of coat proteins and with development of vesicular transport. Clathrin-coated vesicles as vehicle for PS-1 in this area, will be subject of study in further research. In the brainstem, the expression of PS-1 is contrasted completely with the expression of coat proteins, since PS-1 increased progressively from birth until day 15th of age and then decline to values similar to the early ages. We may assume that this phenomenon takes place because PS-1 plays a crucial role in the development of this area during the first two weeks of life. We conclude that, the intracellular transport machinery and endocytic could evolve in line with neurogenesis, suggesting that both routes are essential for neuronal development.

\section{Conflict of interest}

The authors declare that they have no conflict of interest. 


\section{Acknowledgments}

This work was supported by grants from SECTyP (UNCuyoArgentina), the Consejo Nacional de Investigaciones Científicas y Técnicas (CONICET), Argentina, and the VolksWagen Stiftung (Germany). Dr. Miguel Sosa is a Career Researcher of CONICET. We thank Mr. T. Sartor for his valuable technical assistance and Prof. M. Tovar Toulouse for correcting the manuscript.

\section{References}

Abrous, D.N., Koehl, M., Le Moal, M., 2005. Adult neurogenesis: from precursors to network and physiology. Physiol. Rev. 85, 523-569.

Alberdi, A., Sartor, T., Sosa, M.A., 2005. Binding of AP-2 adaptor complex to brain membrane is regulated by phosphorylation of proteins. Biochem. Biophys. Res. Commun. 330, 695-700.

Altman, J., 1982. Morphological development of the rat cerebellum and some of its mechanisms. In: Palay, S.L., Chan-Palay, V. (Eds.), The Cerebellum-New Vistas. Springer, Berlin, pp. 8-49.

Assaife-Lopes, N., Sousa, V.C., Pereira, D.B., Ribeiro, J.A., Chao, M.V., Sebastião, A.M., 2010. Activation of adenosine A2A receptors induces TrkB translocation and increases BDNF-mediated phospho-TrkB localization in lipid rafts: implications for neuromodulation. J. Neurosci. 30, 8468-8480.

Ball, C.L., Hunt, S.P., Robinson, M.S., 1995. Expression and localization of $\alpha$-adaptin isoforms. J. Cell Sci. 108, 2865-2875.

Benzi, G., Moretti, A., 1995. Are reactive oxygen species involved in Alzheimer's disease? Neurobiol. Ageing 16, 661-674.

Blanpied, T.A., Scott, D.B., Ehlers, M.D., 2002. Dynamics and regulation of clathrin coats at specialized endocytic zones of dendrites and spines. Neuron 36, 435-449.

Borgonovo, J., Seltzer, A., Sosa, M.A., 2009. Acute intrastriatal administration of quinolinic acid alters expression and distribution of AP-2 adaptors Possible implications in the endocytotic machinery. J. Neural Transm. 116, 1201-1208.

Bushlin, I., Petralia, R.S., Wu, F., Harel, A., Mughal, M.R., Mattson, M.P., Yao, P.J., 2008 Clathrin assembly protein AP180 and CALM differentially control axogenesis and dendrite outgrowth in embryonic hippocampal neurons. J. Neurosci. 28, 10257-10271.

Chesselet, M.F., Plotkin, J.L., Wu, N., Levine, M.S., 2007. Development of striatal fastspiking GABAergic interneurons. Prog. Brain Res. 160, 261-272.

Collins, B.M., Mc Coy, A.J., Kent, H.M., Evans, P.R., Owe, D.J., 2002. Molecular architecture and functional model of the endocytic AP2 complex. Cell 109, 523-535.

Conner, S.D., Schmidt, S.L., 2003. Regulated portals of entry into the cell. Nature 422 37-44.

Double, K.L., Gerlach, M., Youdim, M.B., Riederer, P., 2000. Impaired iron homeostasis in Parkinson's disease. J. Neural Transm. Suppl. 60, 37-58.

Efthimiopoulos, S., Floor, E., Gerogakopoulos, A., Shioi, J., Cui, W., Yasothornosrikul, S., Hook, V.Y.H., Wisniewski, T., Buee, L., Robakis, N.K., 1998. Enrichment of presenilin 1 peptides in neuronal large dense-core and somatodendritic clathrin-coated vesicles. J. Neurochem. 71, 2365-2372.
Foley, P., Riederer, P., 2000. Influence of neurotoxins and oxidative stress on the onset and progression of Parkinson's disease. J. Neurol. 247, 1182-1194.

Gaidarov, I., Keen, J.H., 1999. Phosphoinositide-AP-2 interactions required for targeting to plasma membrane clathrin-coated pits. J. Cell Biol. 146, 755-764.

Granseth, B., Odermatt, B., Royle, S.J., Lagnado, L., 2007. Clathrin-mediated endocytosis: the physiological mechanism of vesicle retrieval at hippocampal synapses. J. Physiol. 585, 681-686.

Granseth, B., Lagnado, L., 2008. The role of endocytosis in regulating the strength of hippocampal synapses. J. Physiol. 586, 5969-5982.

Halliwell, B., 2001. Role of free radicals in the neurodegenerative diseases therapeutic implications for antioxidant treatment. Drugs Ageing 18, 685-716.

Herbert, V., Shaw, S., Jayantilleke, E., Stopler-Kasdan, T., 1994. Most free-radical injury is iron-related: it is promoted by iron, hemin, holoferritin and vitamin C, and inhibited by desferoxamine and apoferritin. Stem Cells 12, 289-303.

Hines, J.H., Abu-Rub, M., Henley, J.R., 2010. Asymmetric endocytosis and remodeling of beta1-integrin adhesions during growth cone chemorepulsion by MAG. Nat. Neurosci. 13, 829-837.

Hirst, J., Robinson, M.S., 1998. Clathrin and adaptors. Biochim. Biophys. Acta 1404, 173-193.

Hirst, J., Seaman, M.N., Buschow, S.I., Robinson, M.S., 2007. The role of cargo proteins in GGA recruitment. Traffic 8, 594-604.

Jain, M., Armstrong, R.J.E., Barker, R.A., Rosser, A.E., 2001. Cellular and molecular aspects of striatal development. Brain Res. Bull. 55, 533-540.

Jellinger, K.A., 1999. The role of iron in neurodegeneration: prospects for pharmacotherapy of Parkinson's disease. Drugs Ageing 14,115-140.

Kirchhausen, T., 1999. Adaptors for clathrin mediated traffic. Annu. Rev. Cell Dev. Biol. 15, 705-732.

Lowry, O., Rosebrough, N., Farr, A., Randall, R., 1951. Protein measurement with the Folin phenol reagent. J. Biol. Chem. 193, 265-275.

Nagappan, G., Lu, B., 2005. Activity-dependent modulation of the BDNF receptor TrkB: mechanisms and implications. Trends Neurosci. 28, 464-471, Review.

Owen, D.J., Vallis, Y., Noble, M.E., Hunter, J.B., Dafforn, T.R., Evans, P.R., Mc Mahon, H.T., 1999. A structural explanation for the binding of multiple ligands by the $\alpha$-adaptin appendage domain. Cell 97, 805-815.

Ribaut-Barassin, C., Dupont, J.L., Haeberlé, A.M., Bombarde, G., Huber, G., Moussaqui, S., Mariani, J., Bailly, Y., 2003. Alzheimer's disease proteins in cerebellar and hippocampal synapses during postnatal development and aging of the rat. Neuroscience 120, 405-423.

Robinson, M., Bonifacino, J., 2001. Adaptor-related proteins. Curr. Opin. Cell Biol. 13, 444-453.

Samson, F.E., Nelson, S.R., 2000. The aging brain, metals and oxygen free radicals. Cell. Mol. Biol. 46, 699-707.

Wu, F., Yao, P., 2009. Clathrin-mediated endocytosis and Alzheimer's disease: an update. Ageing Res. Rev. 8, 147-149.

Yamashita, T., Eguchi, K., Saitoh, N., von Gersdorff, H., Takahashi, T., 2010. Developmental shift to a mechanism of synaptic vesicle endocytosis requiring nanodomain $\mathrm{Ca}^{2+}$. Nat. Neurosci. 3, 838-844.

Zhang, F., Yim, Y.I., Scarselletta, S., Norton, M., Eisenberg, E., Greene, L.E., 2007. Clathrin adaptor GGA1 polymerizes clathrin into tubules. J. Biol. Chem. 282, 13282-13289. 